\title{
DER JAGDHUND
}

UND DER

\section{FÜNFHUNDERT-ZEHN- wND FÜNFER}

IN DANTE'S COMMEDIA

GEUEUTET YoN

\section{FRIEDRIGH WILH. BERGMANN}

CAV. DELL' ORDINE DEI SANTI MAURIzIO E LAZZARO

MEMBRO DELLA R. COMMISSIONE PE' TEBTI DI LINGUA DI BOLOGNA

SODARE DELL' ACCADEMIA DI SCIENZE LETTERE ED ARTI DE' ZELANTI DI ACI-REALE gOCIO DEI.' ACCADEMIA DI SCIENZE LETTERE ED ARTI DI PALERMO.

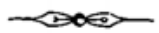

\section{STRASSBURG}

BUCHDRUCKEREI VON G. FISCHBACH

1879 



\author{
SEINEM FREUNDE \\ EDUARD WILH. EUG. REUSS \\ DOCTOR UND PROFESSOR DER THEOLOGIE UND PHILOSOPHIE \\ ETC. ETC. \\ ZUR FEIER SEINES FÜNFZIGJÄHRIGEN LEHRAMTSJUBILÄUMS \\ AM 3 I. JULI 1879 \\ GEWIDMET VON \\ FRIEDRICH WILH. BERGMANN.
}


